In der Rubrik „Literatur kompakt" werden die wichtigsten Originalarbeiten aus der internationalen Fachliteratur referiert.

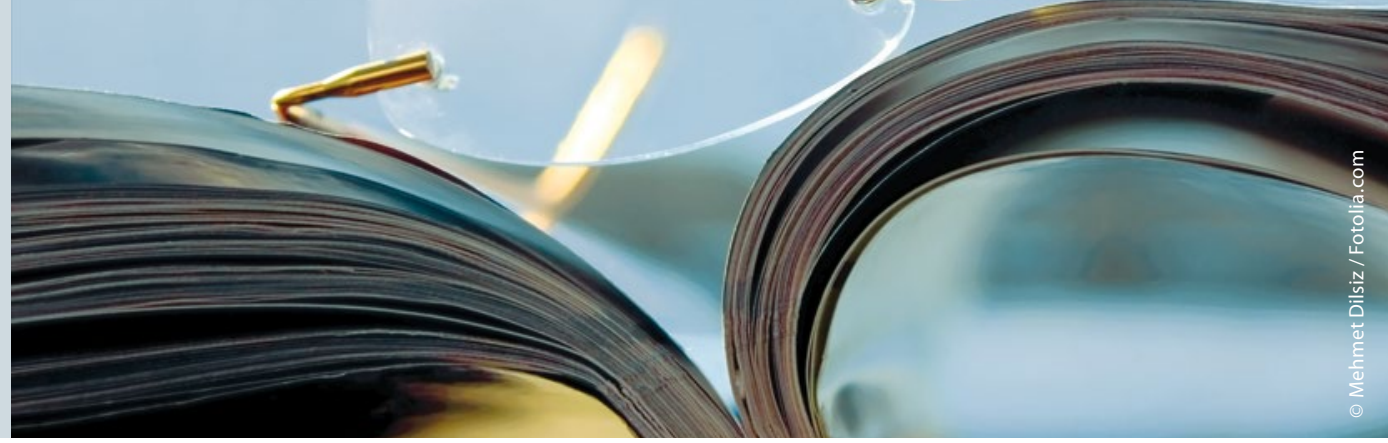

\section{Negative mpMRT schließt signifikantes Karzinom nicht aus}

\author{
Mit der multiparametrischen Magnetresonanztomografie (mpMRT) sollen \\ Patienten mit klinisch irrelevanten Prostatakarzinomen vor einer Über- \\ therapie bewahrt werden. Ob aufgrund einer negativen mpMRT tatsächlich \\ ein signifikantes Prostatakarzinom ausgeschlossen werden kann, haben \\ französische Wissenschaftler in einer retrospektiven Studie untersucht.
}

$\mathrm{D}$ ie mpMRT liefert neben hochauflösenden anatomischen Aufnahmen der Prostataloge auch funktionelle Informationen über Zelldichte, Gefäßversorgung und Durchlässigkeit der Gefäßwände. Anhand dieser Angaben lässt sich beispielsweise ein Diffusionskoeffizient berechnen, der wiederum erlaubt die Dignität einzuschätzen: Krebsgewebe in der Prostata lässt die Diffusion von Wassermolekülen nur eingeschränkt zu.

In der Frühdiagnostik von Prostatakarzinomen setzen viele Experten auf die mpMRT, um Patienten mit klinisch irrelevanten Karzinomen nicht mehr mit Biopsien oder weitergehenden Eingriffen belasten zu müssen. Dies setzt aber voraus, dass negative mpMRT-Befunde zuverlässig einen hohen negativen Vorhersagewert besitzen und falsch-negative Ergebnisse selten sind.

Französische Prostataspezialisten um Nicolas Branger vom Institut Paoli-Calmettes, einem Krebszentrum in Marseille, Frankreich, haben nun in einer retrospektiven Studie geprüf, wie verlässlich negative mpMRT-Resultate tatsächlich sind. Dazu analysierten die Wissenschaftler die Ergebnisse der pathologischen Untersuchung von 101 Prostatektomiepräparaten. Das Gewebe stammte von Männern mit Prostatakarzinomen, die allesamt zuvor eine negative mpMRT-Diagnose erhalten hatten.

Branger und seine Kollegen wollten die Frage klären, wie viele der im mpMRT als negativ beurteilten Vorsteherdrüsen tatsächlich klinisch insignifikante Karzinome bargen - also Karzinome ohne Gleason-Grad 4 oder 5, maximal im Stadium pT2, ohne Lymphknotenbeteiligung und mit einem Volumen von weniger als $0,5 \mathrm{ml}$. Tatsächlich wiesen ein solches insignifikantes Karzinom trotz negativer mpMRT nur $25,3 \%$ der Studienteilnehmer auf. Bei $60,4 \%$ fiel die pathologische Beurteilung ungünstig aus; die Tumoren hatten einen Gleason-Grad von 4 oder 5, es handelte sich um T3-Tumoren oder es waren die Lymphknoten befallen.

Anschließend verglichen Branger und seine Mitarbeiter, wie die Befunde von ausgewiesenen mpMRT-Experten im Vergleich zu Diagnosen anderer Radiologen abschnitten. Negative mpMRTDiagnosen der Experten waren in 29,2 \% der Fälle mit einem insignifikanten Karzinom und in 48,3\% mit einem ungünstigen Tumorprofil verbunden. Damit standen die mpMRT-Experten zwar im direkten Vergleich besser da als ihre nicht spezialisierten Kollegen, deren
Quoten bei 23,6 und 62,5 \% lagen; statistisch signifikant waren die Unterschiede zwischen den beiden Gruppen aber nicht.

Fazit: Branger und seine Kollegen räumen ein, dass es sich bei den untersuchten Männern um ein selektioniertes $\mathrm{Pa}$ tientenkollektiv gehandelt habe. Alle Männer hatten ein Karzinom gehabt und waren aufgrund von klinischen $\mathrm{Pa}$ rametern wie Tastbefund, PSA-Wert oder Biopsieergebnissen, die auf eine signifikante Erkrankung gedeutet hatten, operiert worden. Die Kohorte war also nicht repräsentativ für alle Männer mit Prostatakarzinom und negativem mpMRT-Befund. Das Verhältnis von richtig- und falsch-negativen mpMRTErgebnissen war verzerrt, daher lässt sich aus den vorliegenden Studiendaten von Branger und seinen Kollegen kein gültiger negativer Vorhersagewert berechnen. Eines aber zeige die Untersuchung nach Ansicht der französischen Wissenschaftler gewiss: „Ein negatives mpMRT garantiert nicht, dass kein signifikanter Prostatakrebs vorliegt." Die Rate falsch-negativer Befunde scheine hoch zu sein und es sei unklar, ob eine mpMRT wirklich dabei helfen könne, für oder gegen eine Biopsie oder eine Strategie der aktiven Überwachung zu entscheiden. Qualitätssicherung und Expertise des Radiologen seien dafür jedenfalls wesentliche Voraussetzungen, so Branger et al.

Dr. Robert Bublak

Branger $\mathrm{N}$ et al. Is a negative mpMRI really able to rule out significant prostate cancer? The real life experience. BJU Int. 2016; doi: 10.1111/ bju.13657. 